\title{
Science, interdisciplinarity, and the society
}

\author{
Stephan Lingner
}

Published online: 26 May 2011

(C) Springer-Verlag 2011

Scientific research is organized within a range of different disciplines. Besides some historical contingence, the recent landscape of academics reflects mainly divisions between different objects, terminologies, theories, and methods, which are represented by the specific disciplines. Correspondingly, a huge number of (sub-) disciplines have been established until now. Research within these disciplines has contributed and still contributes to the solution of particular scientific and technical questions for the enhancement of human cognition and related practical capabilities. However, it is imaginable that not every problem will find its appropriate discipline. On the other hand, interdisciplinarity evidently has become a matter of course in modern research; it seems to become the most promising working approach solving the questions of modernity. Both observations may be interrelated: The dimensions of certain contemporary problems address several disciplines at the same time which call for-at least-multi-disciplinary advance. Moreover, some of these overarching problems are even so complex, ambiguous, and uncertain with respect to their consequences that they will need more integrated, interdisciplinary approaches. In some cases, corresponding research may be of explicit societal relevance, thus incorporating trans-disciplinary aims and views.

A prominent example within this exposition is the enterprise of interdisciplinary research on "Global Climate Change" which is of exceptional public interest. Nevertheless, its outcome is often heavily disputed and has therefore to be justified. The expectations of and reasons for respective interdisciplinary research mainly are based on: (1) complex problems of modern societies, (2) huge public funding schemes for certain interdisciplinary research programmes, and (3) controversial debates about the validity of recommendations from interdisciplinary research for decision makers. Against this background, the Europäische Akademie organized a

\footnotetext{
S. Lingner $(\bowtie)$

Europäische Akademie zur Erforschung von Folgen wissenschaftlich-technischer Entwicklungen Bad Neuenahr-Ahrweiler GmbH, Wilhelmstr. 56, 53474 Bad Neuenahr-Ahrweiler, Germany e-mail: stephan.lingner@ea-aw.de
} 
conference on "Interdisciplinary research between societal expectation and scientific validity" last year in Mainz, Germany. Its sessions drew a bow from the state of the sciences over complex problems as reasons for interdisciplinary research to corresponding scientific advice to the society. This journal "Focus" follows that sequence by presenting three papers from the respective sections:

The paper by Eberhard Knobloch (Berlin, Germany) reflects the system of science from a historical perspective by examining "Kaspar Schott's encyclopedia of all mathematical sciences". This encyclopedia was edited at first in 1661 as a textbook for students and scholars and allowed a comprehensive and universal classification of more than twenty scientific disciplines. The underlying concept was realized by means of a set of hierarchical dichotomic criteria which enabled a progressive classification of (sub-)disciplines on successive bifurcation levels. Some of the decisive criteria like "discrete", "abstract" or "concrete" sound familiar even as descriptors of contemporary disciplines. However, in the course of its effort, Schott revised its criteria in order to distinguish "pure" mathematical sciences like geometry from others like astronomy. The latter was subdivided into theoretical and practical efforts. For example, within this scheme, the annual calculation of the Easter event was described as a practical astronomical exercise. Obviously, the classification concept was clearly based on utility questions-however, within a religious context. As a result, it was successfully received by the former scientific community, which demanded for three consecutive editions of this textbook. This historical example reveals the principle and practical value of the disciplinary order of science.

However, disciplinary research mainly pursues scientific long-term programmes, as the next focus author points out. This structure is sometimes inappropriate with regard to today's societal challenges, which often need for prompt, problemspecific, and cross-disciplinary research within temporary projects with foreseeable results. According to the methodological and epistemological reflection of Jan C. Schmidt (Darmstadt, Germany) "On problem-oriented interdisciplinarity", specific demand-driven research forms a constitutive element of this type of interdisciplinarity. This has to be distinguished from other types of interdisciplinarity, which are realized by common objects, theories, and methods. Among the different types of interdisciplinarity, the author stresses the specific societal need and value of problem-oriented research. However, the notion of "problem" remains vague and might be even applied to cognitive problems. In this context, the question "What is a problem?" therefore needs to be clarified. Correspondingly, Schmidt offers a definition that describes a problem as a tension between an inacceptable state of our (social) environment and a desirable target. Transformational knowledge, like that about barriers to be overcome, might add to the comprehensive knowledge on the specific problem. Within this definition, interdisciplinary research turns out to be a necessary service delivery to the society.

Following up, Klaus Mainzer (Munich, Germany) reflects on the "Convergence of research, technology, economy, and society". In its contribution "interdisciplinarity and innovation dynamics", he assumes innovation as a key for national welfare in a globalized world which could be seen as a desirable societal goal in the above-mentioned sense. In modernity, innovation is the result of problem-oriented 
research without limits of traditional disciplinary borders. Instead, corresponding interdisciplinary research on societal questions and new technological options will focus the different professional perspectives to the scientific problem. The clustering and integration of the relevant disciplines might be organized or even institutionalized as forums of tight interdisciplinary communication, as it was demonstrated within the German "Excellence Initiative". The author proposes to accompany this focused interdisciplinary research by related academic training programmes. Corresponding added value is for instance generated by the Carl von Linde-Academy at the Technical University of Munich. While conducting interdisciplinarity this way, the academy considers itself to continue the humanistic tradition of antiquity.

Concluding, interdisciplinary research has a specific potential and power for the solution of ambitious, difficult, uncertain, and ambivalent questions with societal relevance. However, that does not mean that interdisciplinarity would be prudent or necessary in each case. Moreover, one should also refrain from using interdisciplinarity as a "buzz word", in order to prevent a drain of its meaning. Instead and in light of successful disciplinary research, interdisciplinary projects have always to substantiate their claims for problem-related appropriateness. 